\title{
Review of: "Systemic and local immune response to intraocular AAV vector administration in non-human primates"
}

Hildegund Ertl

Potential competing interests: The author(s) declared that no potential competing interests exist.

The manuscript describes antibody responses to AAV in non-human primates that received ocular AAV mediated gene transfer and correlated antibody responses with intraocular inflammatory responses.

Overall the. results are predictive and thereby not novel. Injection of a low dose of an AAV vector does not increase antibody titers to AAV while higher doses lead to increases. Antibody titers are sustained as has been described previously and cross-react between different AAV serotypes, which is also known. As one would expect lower doses did not lead to significant local pathology, while medium and higher doses did. Increases in AAV-specific antibodies correlated with severity of the inflammatory response within the eye which again would be expected as the inflammatory responses initiated activation of adaptive immune responses.

Overall, although the manuscript has some interesting data that may aid the design of clinical trials that use ocular transfer of AAV vectors, a shorted and more concise version without data that simply confirm the finding of others would be useful. 